\title{
On the remainder term in the circle problem in an arithmetic progression
}

\author{
D. I. Tolev* \\ Dedicated to 75 th birthday of Professor A. A. Karatsuba
}

\begin{abstract}
In this paper we improve the estimate for the remainder term in the asymptotic formula concerning the circle problem in an arithmetic progression.

Keywords: Circle problem, Arithmetic progressions.

Mathematics Subject Classification (2010): 11L05, 11 N37.
\end{abstract}

\section{Introduction and statement of the result.}

Consider the sum

$$
S_{q, a}(x)=\sum_{\substack{n \leq x \\ n \equiv a}} r(n),
$$

where $r(n)$ is the number of representations of $n$ as a sum of two squares. In 1968 Smith 9 ] established that if $q=O\left(x^{\frac{2}{3}}\right)$ then we have the following asymptotic formula

$$
S_{q, a}(x)=\pi \frac{\eta_{a}(q)}{q^{2}} x+R_{q, a}(x) .
$$

The quantity $\eta_{a}(q)$ in the main term is defined by

$$
\eta_{a}(q)=\#\left\{1 \leq \alpha, \beta \leq q: \alpha^{2}+\beta^{2} \equiv a \quad(\bmod q)\right\}
$$

(the main term in Smith's paper is written in a slightly different form) and $R_{q, a}(x)$ is the remainder term for which it is established that

$$
R_{q, a}(x) \ll x^{\frac{2}{3}+\xi} q^{-\frac{1}{2}(1+3 \xi)}(q, a)^{\frac{1}{2}} \tau(q) .
$$

\footnotetext{
*Supported by the Ministry of Science and Education of Bulgaria, Grant DD VU 02/90.
} 
Here $\tau(q)$ is the divisor function, $(q, a)$ stands for the greatest common divisor of $a$ and $q$ and $0<\xi<\frac{1}{3}$.

We note that uniformly for $a$ we have

$$
\eta_{a}(q) \ll q \tau(q)
$$

(a proof is available in [3, Lemma 2.8]) and obviously $S_{q, a}(x) \ll x^{1+\varepsilon} q^{-1}$ for any $\varepsilon>0$. Hence the asymptotic formula (21) is non-trivial for $q \leq x^{\frac{2}{3}-\varepsilon}$. However if $q$ is small then the estimate (4) for the error term is quite weak.

In 1970 Varbanets [11] 1 considered the case $a=1$ and found better estimates for the remainder term in (2). He established that if $x^{\frac{1}{2}} \leq q \leq x^{\frac{2}{3}}$ then

$$
R_{q, 1}(x) \ll\left(q^{\frac{1}{2}}+x^{\frac{1}{2}} q^{-\frac{1}{4}}\right) x^{\varepsilon} .
$$

Varbanets also proved that if $q \leq x^{\frac{2}{3}}$ and

$$
q^{\frac{1}{4}}>\xi(q), \quad \text { where } \quad \xi(q)=\prod_{p \mid q} p
$$

(the product is taken over the prime divisors of $q$ ), then

$$
R_{q, 1}(x) \ll\left(q^{\frac{1}{2}}+x^{\frac{1}{2}} q^{-\frac{1}{2}} \xi(q)\right) x^{\varepsilon} .
$$

(The results of [11] are actually slightly stronger — with a power of $\tau(q)$ rather than $x^{\varepsilon}$ ).

It is clear that our sum $S_{q, a}(x)$ is very similar to

$$
T_{q, a}(x)=\sum_{\substack{n \leq x \\ n \equiv a}} \tau(n) .
$$

An asymptotic formula for $T_{q, a}(x)$, which is non-trivial for $q \leq x^{\frac{2}{3}-\varepsilon}$, was established independently by A. Selberg and C. Hooley (in unpublished manuscripts). It is well known today that if $(q, a)=1$ then we have

$$
T_{q, a}(x)=\frac{\varphi(q)}{q^{2}} x\left(\log x+2 \gamma-1+2 \sum_{p \mid q} \frac{\log p}{p-1}\right)+O\left(\tau^{2}(q)\left(q^{\frac{1}{2}}+x^{\frac{1}{3}}\right) \log x\right),
$$

\footnotetext{
${ }^{1}$ The author would like to thank Mr Ping Xi for informing him about this paper.
} 
where $\varphi(q)$ is the Euler function, $\gamma$ stands for the Euler constant and where the summation is taken over the prime divisors of $q$. A proof of (9) can be found for example in a recent paper of Blomer [2] or in the book of Iwaniec and Kowalski [8, Chapter 4]. One may expect that a formula similar to (9) holds also for the sum $S_{q, a}(x)$.

In the present paper we prove the following:

Theorem. For the quantity $R_{q, a}(x)$ defined by (2) we have

$$
R_{q, a}(x) \ll\left(q^{\frac{1}{2}}+x^{\frac{1}{3}}\right)(a, q)^{\frac{1}{2}} \tau^{4}(q) \log ^{4} x .
$$

The asymptotic formula (2) with the estimate (10) for the remainder term is non-trivial for $q \leq x^{\frac{2}{3}-\varepsilon}$ and (10) is stronger than (6) for all such $q$. If we compare our bound for $R_{q, a}(x)$ with (8), which is established only for $a=1$ and for $q$ satisfying (17), we can see that (10) is stronger provided that $q \ll x^{\frac{1}{3}} \xi(q)^{2}$.

One of the main points in our proof is the estimation of the sum $\mathcal{H}_{h, n}(q, a)$ defined by (44). In Section 2.3 we represent it as a linear combination of Kloosterman sums and then apply A. Weil's bound. An estimate of the same strength for $\mathcal{H}$ in the case $a=1$ is established in [11] but appealing to a result of Bombieri [1]. It is clear that Varbanets' method can be applied also for the estimation of $\mathcal{H}$ for any integer $a$, but here we present our method, which may be of some use in other occasions. The other arguments of the proof are elementary or based on the simplest theorems from the theory of the exponential sums.

Working in the same manner one may find another proof of (9) and one may also establish similar asymptotic formulas for the quantities

$$
\sum_{\substack{m^{2}+n^{2} \leq x \\ m n \equiv a(q)}} 1 \quad \text { and } \quad \sum_{\substack{m n \leq x \\ m^{2}+n^{2} \equiv a(q)}} 1
$$

(here and later we write for simplicity $k \equiv a(q)$ instead of $k \equiv a(\bmod q)$ ). Such kind of problems have been already considered by several authors - we refer the reader to a recent paper of Ustinov [10], for example, where references to other papers can be found. However we will not discuss these more general problems here. 


\section{Proof of the theorem}

\subsection{Preparation}

We assume that $q \leq x^{\frac{2}{3}}$ because otherwise formula (10) is trivial.

The sum $S_{q, a}(x)$ defined by (1) is obviously equal to the number of pairs of integers $u, v$ satisfying

$$
u^{2}+v^{2} \leq x, \quad u^{2}+v^{2} \equiv a(q)
$$

Therefore

$$
S_{q, a}(x)=4 S^{\prime}+4 S^{\prime \prime}+O(1)
$$

where $S^{\prime}$ is the number of pairs of positive integers $u, v$ satisfying (11) and respectively $S^{\prime \prime}$ is the number of positive integers $u \leq \sqrt{x}$ such that

$$
u^{2} \equiv a(q)
$$

(From this point onwards by $u$ and $v$ we denote natural numbers only).

It is clear that

$$
S^{\prime \prime}=\frac{\omega_{a}(q)}{q} \sqrt{x}+O\left(\omega_{a}(q)\right)
$$

where

$$
\omega_{a}(q)=\#\left\{1 \leq \alpha \leq q: \alpha^{2} \equiv a(q)\right\}
$$

We note that this function satisfies

$$
\omega_{a}(q) \ll(q, a)^{\frac{1}{2}} \tau(q) .
$$

This can be proved in a simple elementary way and we leave the verification to the reader.

The sum $S^{\prime}$ can be written in the form

$$
S^{\prime}=2 S_{1}-S_{2}
$$

Here $S_{1}$ is the number of pairs of natural numbers $u, v$ satisfying the congruence

$$
u^{2}+v^{2} \equiv a(q)
$$

and such that

$$
u \leq \sqrt{x / 2}, \quad v \leq \sqrt{x-u^{2}}
$$


Respectively $S_{2}$ is the number pairs $u, v$ satisfying (18) and also

$$
u \leq \sqrt{x / 2}, \quad v \leq \sqrt{x / 2}
$$

We divide $S_{1}$ and $S_{2}$ into parts according to the congruence classes of $u$ and $v$ modulo $q$. From this point we write for simplicity

$$
\sum_{\alpha, \beta} \text { for } \sum_{\substack{1 \leq \alpha, \beta \leq q \\ \alpha^{2}+\beta^{2} \equiv a(q)}}
$$

In this notation we have

$$
S_{1}=\sum_{\alpha, \beta} G_{1}(\alpha, \beta), \quad S_{2}=\sum_{\alpha, \beta} G_{2}(\alpha, \beta)
$$

where $G_{1}(\alpha, \beta)$ is the number or pairs $u, v$ satisfying (19) and also

$$
u \equiv \alpha(q), \quad v \equiv \beta(q)
$$

and respectively $G_{2}(\alpha, \beta)$ is the number of pairs $u, v$ with (20) and (23).

Consider $G_{1}(\alpha, \beta)$. Denote as usual by $[y]$ and $\{y\}$ the integer part and the fractional part of $y$ and let

$$
\rho(y)=\frac{1}{2}-\{y\}
$$

We use that for any $y \geq 0$ we have

$$
\sum_{\substack{u \leq y \\ u \equiv \gamma(q)}} 1=\left[\frac{y-\gamma}{q}\right]-\left[\frac{-\gamma}{q}\right]=\frac{y}{q}+\rho\left(\frac{y-\gamma}{q}\right)-\rho\left(\frac{-\gamma}{q}\right) .
$$

Hence for any $u$ satisfying the first condition in (19) the number of integers $v$ satisfying the second of these conditions and also the second congruence from (23) equals

$$
\frac{\sqrt{x-u^{2}}}{q}+\rho\left(\frac{\sqrt{x-u^{2}}-\beta}{q}\right)-\rho\left(\frac{-\beta}{q}\right) .
$$

Therefore

$$
G_{1}(\alpha, \beta)=\frac{1}{q} \sum_{\substack{u \leq \sqrt{x / 2} \\ u \equiv \alpha(q)}} \sqrt{x-u^{2}}+\sum_{\substack{u \leq \sqrt{x / 2} \\ u \equiv \alpha(q)}} \rho\left(\frac{\sqrt{x-u^{2}}-\beta}{q}\right)-\sum_{\substack{u \leq \sqrt{x / 2} \\ u \equiv \alpha(q)}} \rho\left(\frac{-\beta}{q}\right) .
$$


We substitute this expression for $G_{1}$ in the first formula in (22) and we get

$$
S_{1}=\frac{1}{q} S_{1}^{(0)}+S_{1}^{(1)}-S_{1}^{(2)}
$$

where

$$
\begin{aligned}
& S_{1}^{(0)}=\sum_{\alpha, \beta} \sum_{\substack{u \leq \sqrt{x / 2} \\
u \equiv \alpha(q)}} \sqrt{x-u^{2}}, \\
& S_{1}^{(1)}=\sum_{\alpha, \beta} \sum_{\substack{u \leq \sqrt{x / 2} \\
u \equiv \alpha(q)}} \rho\left(\frac{\sqrt{x-u^{2}}-\beta}{q}\right), \\
& S_{1}^{(2)}=\sum_{\alpha, \beta} \sum_{\substack{u \leq \sqrt{x / 2} \\
u \equiv \alpha(q)}} \rho\left(\frac{-\beta}{q}\right) .
\end{aligned}
$$

We proceed with $G_{2}$ in the same manner and using the second formula in (22) we get

$$
S_{2}=\frac{\sqrt{x / 2}}{q} S_{2}^{(0)}+S_{2}^{(1)}-S_{1}^{(2)}
$$

where $S_{1}^{(2)}$ is specified by (29) and

$$
\begin{aligned}
S_{2}^{(0)} & =\sum_{\alpha, \beta} \sum_{\substack{u \leq \sqrt{x / 2} \\
u \equiv \alpha(q)}} 1, \\
S_{2}^{(1)} & =\sum_{\alpha, \beta} \sum_{\substack{u \leq \sqrt{x / 2} \\
u \equiv \alpha(q)}} \rho\left(\frac{\sqrt{x / 2}-\beta}{q}\right) .
\end{aligned}
$$

From (12), (14), (16), (17), (26) and (30) we obtain

$$
\begin{aligned}
S_{q, a}(x)=\frac{8}{q} S_{1}^{(0)} & +8 S_{1}^{(1)}-4 S_{1}^{(2)}-4 \frac{\sqrt{x / 2}}{q} S_{2}^{(0)} \\
& -4 S_{2}^{(1)}+4 \frac{\omega_{a}(q)}{q} \sqrt{x}+O\left((q, a)^{\frac{1}{2}} \tau(q)\right) .
\end{aligned}
$$


We estimate the sum $S_{1}^{(1)}$ in sections 2.2 - 2.5. This is the most difficult part of the proof. In section 2.6 we evaluate $S_{1}^{(0)}, S_{1}^{(2)}, S_{2}^{(0)}$ and $S_{2}^{(1)}$. Finally in section 2.7 we collect all results together and prove the asymptotic formula (10).

\subsection{Estimation of $S_{1}^{(1)}$ - beginning.}

It is well known that for any integer $M \geq 2$ the function $\rho(y)$ defined by (24) can be written in the form

$$
\rho(y)=\sum_{1 \leq|n| \leq M} \frac{e(n y)}{2 \pi i n}+O\left(\min \left(1, \frac{1}{M\|y\|}\right)\right),
$$

where $\|y\|$ is the distance from $y$ to the nearest integer, $e(y)=e^{2 \pi i y}$, the constant in the $O$-symbol is absolute and where, as usual, we assume that $\min \left(1, \frac{1}{0}\right)=1$. We also have

$$
\min \left(1, \frac{1}{M\|y\|}\right)=\sum_{n \in \mathbb{Z}} c_{n} e(n y)
$$

where

$$
c_{n} \ll \begin{cases}M^{-1} \log M & \text { for all } n, \\ M n^{-2} & \text { for } n \neq 0 .\end{cases}
$$

For the proofs of (34) - (36) we refer the reader to [6, Chapter 2].

We take an integer $M \geq 2$, which we shall choose later, and using (28), (34) we obtain

$$
S_{1}^{(1)}=\sum_{\alpha, \beta} \sum_{\substack{u \leq \sqrt{x / 2} \\ u \equiv \alpha(q)}} \sum_{\substack{1 \leq|n| \leq M \\ 2 \pi i n}} \frac{1}{2 \pi}\left(\frac{\sqrt{x-u^{2}}-\beta}{q} n\right)+O(\Delta),
$$

where

$$
\Delta=\sum_{\alpha, \beta} \sum_{\substack{u \leq \sqrt{x / 2} \\ u \equiv \alpha(q)}} \min \left(1, M^{-1}\left\|\frac{\sqrt{x-u^{2}}-\beta}{q}\right\|^{-1}\right) .
$$

Now we apply (35) to get

$$
\Delta=\sum_{\alpha, \beta} \sum_{\substack{u \leq \sqrt{x / 2} \\ u \equiv \alpha(q)}} \sum_{n \in \mathbb{Z}} c_{n} e\left(\frac{\sqrt{x-u^{2}}-\beta}{q} n\right)=\sum_{n \in \mathbb{Z}} c_{n} \mathcal{F}_{n}
$$


where

$$
\mathcal{F}_{n}=\sum_{\alpha, \beta} \sum_{\substack{u \leq \sqrt{x / 2} \\ u \equiv \alpha(q)}} e\left(\frac{\sqrt{x-u^{2}}-\beta}{q} n\right) .
$$

From (36), (37), (39) and (401) we find

$$
S_{1}^{(1)} \ll \frac{\log M}{M}\left|\mathcal{F}_{0}\right|+\log M \sum_{1 \leq|n| \leq M}|n|^{-1}\left|\mathcal{F}_{n}\right|+M \sum_{|n|>M} n^{-2}\left|\mathcal{F}_{n}\right| .
$$

Consider the sum $\mathcal{F}_{n}$. We use the elementary identity

$$
\sum_{h(q)} e\left(\frac{h m}{q}\right)= \begin{cases}q & \text { for } q \mid m, \\ 0 & \text { otherwise }\end{cases}
$$

(the summation is taken over all residue classes $h(\bmod q)$ ) and we find

$$
\begin{aligned}
\mathcal{F}_{n} & =\sum_{\alpha, \beta} \sum_{u \leq \sqrt{x / 2}} e\left(\frac{\sqrt{x-u^{2}}-\beta}{q} n\right) \frac{1}{q} \sum_{h(q)} e\left(\frac{h(u-\alpha)}{q}\right) \\
& =\frac{1}{q} \sum_{h(q)} \mathcal{H}_{h, n} \mathcal{T}_{h, n},
\end{aligned}
$$

where

$$
\begin{aligned}
\mathcal{H}_{h, n} & =\mathcal{H}_{h, n}(q, a)=\sum_{\alpha, \beta} e\left(\frac{-\alpha h-\beta n}{q}\right), \\
\mathcal{T}_{h, n} & =\mathcal{T}_{h, n}(q)=\sum_{u \leq \sqrt{x / 2}} e(f(u)), \\
f(u) & =\left(n \sqrt{x-u^{2}}+h u\right) q^{-1} .
\end{aligned}
$$

From (43) we obtain

$$
\left|\mathcal{F}_{n}\right| \leq \frac{1}{q} \sum_{|h| \leq q / 2}\left|\mathcal{H}_{h, n}\right|\left|\mathcal{T}_{h, n}\right|
$$

so to proceed further we have to estimate the sums $\mathcal{H}_{h, n}$ and $\mathcal{T}_{h, n}$. 


\subsection{Estimation of $\mathcal{H}_{h, n}$.}

\subsubsection{Preparation.}

In section 2.3 we establish that

$$
\left|\mathcal{H}_{h, n}(q, a)\right| \leq 4 q^{\frac{1}{2}} \tau^{2}(q)(q, h, n)^{\frac{1}{2}}\left(q, a, h^{2}+n^{2}\right)^{\frac{1}{2}}
$$

where as usual $(u, v, w)$ is the greatest common divisor of $u, v, w$. We note that in the proof of (10) we actually use only the following consequence of (48):

$$
\left|\mathcal{H}_{h, n}(q, a)\right| \leq 4 q^{\frac{1}{2}} \tau^{2}(q)(q, h, n)^{\frac{1}{2}}(q, a)^{\frac{1}{2}} .
$$

To establish (48) we first express $\mathcal{H}_{h, n}$ by means of the Gauss sum

$$
S(q ; k, m)=\sum_{\alpha(q)} e\left(\frac{k \alpha^{2}+m \alpha}{q}\right)
$$

Using (21), (42) and (44) we get

$$
\begin{aligned}
\mathcal{H}_{h, n}(q, a) & =\sum_{\alpha, \beta} e\left(\frac{\alpha h+\beta n}{q}\right)=\sum_{\alpha(q)} \sum_{\beta(q)} e\left(\frac{\alpha h+\beta n}{q}\right) \frac{1}{q} \sum_{k(q)} e\left(\frac{k\left(\alpha^{2}+\beta^{2}-a\right)}{q}\right) \\
& =\frac{1}{q} \sum_{k(q)} e\left(\frac{-a k}{q}\right) S(q ; k, h) S(q ; k, n) .
\end{aligned}
$$

We know that

$$
S(q ; k, n)=\left\{\begin{array}{ll}
d S(q / d ; k / d, n / d) & \text { for } d \mid n, \\
0 & \text { for } d \nmid n .
\end{array} \text { where } d=(k, q),\right.
$$

A proof of this relation can be found in [4, Section 6]. Therefore from (51) we find

$$
\begin{aligned}
\mathcal{H}_{h, n}(q, a) & =\frac{1}{q} \sum_{d \mid q} \sum_{\substack{k(q) \\
(k, q)=\frac{q}{d}}} e\left(\frac{-a k}{q}\right) S(q ; k, h) S(q ; k, n) \\
& =q \sum_{\substack{d\left|q \\
\frac{q}{d}\right|(h, n)}} d^{-2} B(d ; a, h d / q, n d / q),
\end{aligned}
$$


where

$$
B(d ; a, m, t)=\sum_{l(d)}^{*} e\left(\frac{-a l}{d}\right) S(d ; l, m) S(d ; l, t)
$$

(the asterisk means that the summation is restricted to a reduced system of residues).

Next we use other properties of the Gauss sum to represent the quantity $B$ by means of the Kloosterman sum

$$
K(q ; k, n)=\sum_{\alpha(q)}^{*} e\left(\frac{k \alpha+n \bar{\alpha}}{q}\right),
$$

where $\bar{\alpha}$ is the inverse of $\alpha$ modulo $q$, and then we apply A.Weil's bound

$$
|K(q ; k, n)| \leq q^{\frac{1}{2}} \tau(q)(q, k, n)^{\frac{1}{2}} .
$$

A proof of (56) is available in [8, Chapter 11].

The calculations are simpler if $q$ is odd but certain technical complications arise in the general case. That is why we consider separately the cases $2 \nmid q$ and $q=2^{\theta}$ and establish corresponding versions of (48). Finally we note that $\mathcal{H}_{h, n}(q, a)$ has a multiplicative property with respect to $q$ and prove (48).

\subsubsection{The case $2 \nmid q$.}

In this section we prove that

$$
\left|\mathcal{H}_{h, n}(q, a)\right| \leq q^{\frac{1}{2}} \tau^{2}(q)(q, h, n)^{\frac{1}{2}}\left(q, a, h^{2}+n^{2}\right)^{\frac{1}{2}} \quad \text { for } \quad 2 \nmid q .
$$

Having in mind (53) we see that we have to estimate the quantity $B$ defined by (54) for any $d \mid q$.

We first note that the Gauss sum satisfies

$$
S(q ; k, m)=e\left(\frac{-\overline{(4 k)} m^{2}}{q}\right)\left(\frac{k}{q}\right) S(q ; 1) \quad \text { for } \quad(q, 2 k)=1,
$$

where $\left(\frac{k}{q}\right)$ is the Jacobi symbol and $S(q ; 1)=S(q ; 1,0)$. A proof of (158) can be found in [4, Section 6]. Using (54), (55), (58) and bearing in mind that $2 \nmid d$ for any $d \mid q$ we get

$$
B(d ; a, m, t)=S^{2}(d ; 1) K\left(d ; a, \overline{4}\left(m^{2}+t^{2}\right)\right),
$$


where $\overline{4}$ is the inverse of 4 modulo $d$. It is well known that

$$
|S(d ; 1)|^{2}=d \quad \text { for } \quad 2 \nmid d
$$

(a proof is available for example [7, Chapter 7]). From this formula and (56) we find

$$
|B(d ; a, m, t)| \leq d^{\frac{3}{2}} \tau(d)\left(d, a, m^{2}+t^{2}\right)^{\frac{1}{2}} .
$$

We substitute this bound for $B$ in (53) (with $m=h d / q$ and $t=n d / q$ ) to get

$$
\left|\mathcal{H}_{h, n}(q, a)\right| \leq q \tau(q)\left(q, a, h^{2}+n^{2}\right)^{\frac{1}{2}} \mathfrak{X}, \quad \mathfrak{X}=\sum_{\substack{d\left|q \\ \frac{q}{d}\right|(h, n)}} d^{-\frac{1}{2}} .
$$

It is obvious that

$$
\mathfrak{X}=\sum_{\delta \mid(q, h, n)}\left(\frac{q}{\delta}\right)^{-\frac{1}{2}} \leq q^{-\frac{1}{2}} \tau(q)(q, h, n)^{\frac{1}{2}}
$$

hence we obtain (57).

\subsubsection{The case $q=2^{\theta}$.}

In this section we prove that

$$
\left|\mathcal{H}_{h, n}\left(2^{\theta}, a\right)\right| \leq 4\left(2^{\theta}\right)^{\frac{1}{2}} \tau^{2}\left(2^{\theta}\right)\left(2^{\theta}, n, h\right)^{\frac{1}{2}}\left(2^{\theta}, a, h^{2}+n^{2}\right)^{\frac{1}{2}} .
$$

First we establish that the sum $B$ given by (54) satisfies

$$
\left|B\left(2^{\nu}, a, m, t\right)\right| \leq 4\left(2^{\nu}\right)^{\frac{3}{2}} \tau\left(2^{\nu}\right)\left(2^{\nu}, a, m^{2}+t^{2}\right)^{\frac{1}{2}} .
$$

The inequality (60) is obvious for $\nu<2$, so we may assume that $\nu \geq 2$. Under this assumption and if $2 \nmid r$ the Gauss sum satisfies

$$
S\left(2^{\nu}, r, t\right)=\left\{\begin{array}{lll}
e\left(\frac{-\bar{r}(t / 2)^{2}}{2^{\nu}}\right) 2^{\frac{\nu+1}{2}} \frac{1+i^{r}}{\sqrt{2}}, & \text { for } 2|t, \quad 2| \nu, \\
e\left(\frac{-\bar{r}(t / 2)^{2}}{2^{2}}\right) 2^{\frac{\nu+1}{2}} e\left(\frac{r}{8}\right), & \text { for } 2 \mid t, & 2 \nmid \nu, \\
0 & \text { for } 2 \nmid t .
\end{array}\right.
$$

For a proof we refer the reader to [4, Section 6] and [7, Chapter 7].

From (54) and (61) it follows that (60) holds if $2 \nmid m$ or $2 \nmid t$. 
Consider now the case when $2 \mid m$ and $2 \mid t$. If we have also $2 \mid \nu$ then (54) and (61) imply

$$
B\left(2^{\nu}, a, m, t\right)=i 2^{\nu+1} \sum_{l\left(2^{\nu}\right)}^{*}(-1)^{\frac{l-1}{2}} e\left(\frac{-l a-\bar{l}\left(\left(\frac{m}{2}\right)^{2}+\left(\frac{t}{2}\right)^{2}\right)}{2^{\nu}}\right)
$$

and having in mind (55) we get

$$
B\left(2^{\nu}, a, m, t\right)=2^{\nu+1} K\left(2^{\nu} ; 2^{\nu-2}-a,-\frac{m^{2}+t^{2}}{4}\right) .
$$

Now we apply (56) and obtain

$$
\left|B\left(2^{\nu}, a, m, t\right)\right| \leq 2\left(2^{\nu}\right)^{\frac{3}{2}} \tau\left(2^{\nu}\right)\left(2^{\nu}, 2^{\nu-2}-a, \frac{m^{2}+t^{2}}{4}\right)^{\frac{1}{2}},
$$

which implies (60).

If $2 \nmid \nu$ then using (54), (55) and (61) we see that (62) is true again, hence (60) holds also in this case.

Now we proceed as in section 2.3 .2 and using (60) we obtain (159).

\subsubsection{The estimate for $\mathcal{H}_{h, n}$.}

We are now in a position to prove (48) in the general case. We have the following identity

$$
\mathcal{H}_{h, n}\left(q_{1} q_{2}, a\right)=\mathcal{H}_{h \overline{q_{2}}, n \overline{q_{2}}}\left(q_{1}, a\right) \mathcal{H}_{h \overline{q_{1}}, n \overline{q_{1}}}\left(q_{2}, a\right), \quad \text { for } \quad\left(q_{1}, q_{2}\right)=1
$$

where $\overline{q_{2}}$ and $\overline{q_{1}}$ denote the inverses of $q_{2}$ and $q_{1}$ modulo $q_{1}$ and respectively $q_{2}$. The proof of (63) is standard and we leave it to the reader. Now we represent $q=q_{1} q_{2}$, where $2 \nmid q_{1}$ and $q_{2}=2^{\theta}$, then we apply (57), (59) and (하) and obtain (48).

\subsection{Estimation of $\mathcal{T}_{h, n}$ and $\mathcal{F}_{n}$.}

\subsubsection{The sum $\mathcal{T}_{h, n}$.}

In this section we assume that $|h| \leq \frac{q}{2}$ and establish that

$$
\mathcal{T}_{h, n} \ll \min \left(\sqrt{x}, q|h|^{-1}\right) \quad \text { if } \quad n=0 \quad \text { or } \quad 0<2|n| \leq|h|
$$

and

$$
\mathcal{T}_{h, n} \ll x^{\frac{1}{4}}\left(|n|^{\frac{1}{2}} q^{-\frac{1}{2}}+|n|^{-\frac{1}{2}} q^{\frac{1}{2}}\right) \quad \text { for } \quad n \neq 0 .
$$


If $n=0$ then the inequality (64) follows from the well-known estimate for linear exponentials sums (see [5, Chapter 2.1]).

Suppose now that $n \neq 0$. The function $f(u)$ defined by (46) satisfies

$$
f^{\prime}(u)=\left(-n u\left(x-u^{2}\right)^{-\frac{1}{2}}+h\right) q^{-1}, \quad f^{\prime \prime}(u)=-n x\left(x-u^{2}\right)^{-\frac{3}{2}} q^{-1} .
$$

In the case $0<2|n| \leq|h| \leq \frac{q}{2}$ we use the first formula and find

$$
\frac{|h|}{2 q} \leq\left|f^{\prime}(u)\right| \leq \frac{3|h|}{2 q} \leq \frac{3}{4} \quad \text { for } \quad 0 \leq u \leq \sqrt{x / 2} .
$$

Then we apply [5, Theorem 2.1] and we obtain (64).

Further, it is clear that

$$
\left|f^{\prime \prime}(u)\right| \asymp|n| q^{-1} x^{-\frac{1}{2}} \quad \text { for } \quad 0 \leq u \leq \sqrt{x / 2} .
$$

Now we apply [5, Theorem 2.2] and find

$$
\mathcal{T}_{n, h} \ll x^{\frac{1}{2}}\left(|n| q^{-1} x^{-\frac{1}{2}}\right)^{\frac{1}{2}}+\left(|n| q^{-1} x^{-\frac{1}{2}}\right)^{-\frac{1}{2}},
$$

which gives (65).

\subsubsection{The $\operatorname{sum} \mathcal{F}_{n}$.}

If $n=0$ then we apply (47), (49) and (64) to get

$$
\begin{aligned}
\mathcal{F}_{0} & \ll q^{-\frac{1}{2}} \tau^{2}(q)(q, a)^{\frac{1}{2}} \sum_{|h| \leq \frac{q}{2}}(q, h)^{\frac{1}{2}} \min \left(\sqrt{x}, q|h|^{-1}\right) \\
& \ll \tau^{2}(q)(q, a)^{\frac{1}{2}}\left(x^{\frac{1}{2}}+q^{\frac{1}{2}} \sum_{1 \leq h \leq q} \frac{(q, h)^{\frac{1}{2}}}{h}\right) .
\end{aligned}
$$

However for any $y \geq 2$ we have

$$
\sum_{n \leq y} \frac{(q, n)^{\frac{1}{2}}}{n} \leq \sum_{\delta \mid q} \delta^{\frac{1}{2}} \sum_{\substack{n \leq y \\ n \equiv 0(\delta)}} \frac{1}{n} \leq \sum_{\delta \mid q} \delta^{-\frac{1}{2}} \sum_{n \leq \frac{y}{\delta}} \frac{1}{n} \ll \tau(q) \log y,
$$

hence we get

$$
\mathcal{F}_{0} \ll x^{\frac{1}{2}} \tau^{3}(q)(q, a)^{\frac{1}{2}} \log x
$$


In the case $n \neq 0$ we apply (47) and (49) to find

$$
\mathcal{F}_{n} \ll q^{-\frac{1}{2}} \tau^{2}(q)(q, a)^{\frac{1}{2}}(q, n)^{\frac{1}{2}} \mathfrak{T}_{n}, \quad \mathfrak{T}_{n}=\sum_{|h| \leq \frac{q}{2}}\left|\mathcal{T}_{h, n}\right| .
$$

However from (64) and (65)) it follows that

$$
\mathfrak{T}_{n} \ll \sum_{2|n| \leq|h| \leq \frac{q}{2}} q|h|^{-1}+\sum_{|h| \leq \min \left(2|n|, \frac{q}{2}\right)} x^{\frac{1}{4}}\left(|n|^{\frac{1}{2}} q^{-\frac{1}{2}}+|n|^{-\frac{1}{2}} q^{\frac{1}{2}}\right) \ll q \log x+x^{\frac{1}{4}}|n|^{\frac{1}{2}} q^{\frac{1}{2}}
$$

and we get

$$
\mathcal{F}_{n} \ll\left(q^{\frac{1}{2}}+x^{\frac{1}{4}}|n|^{\frac{1}{2}}\right) \tau^{2}(q)(q, a)^{\frac{1}{2}}(q, n)^{\frac{1}{2}} \log x \quad \text { for } \quad n \neq 0 .
$$

\subsection{Estimation of $S_{1}^{(1)}$ - end.}

We use (41), (67) and (68) to find

$$
\begin{gathered}
\left.S_{1}^{(1) \ll\left(x^{\frac{1}{2}}\right.} M^{-1}+\left(q^{\frac{1}{2}}+x^{\frac{1}{4}} M^{\frac{1}{2}}\right) \sum_{1 \leq n \leq M} \frac{(q, n)^{\frac{1}{2}}}{n}+M \sum_{n>M} \frac{(q, n)^{\frac{1}{2}}}{n^{2}}\left(q^{\frac{1}{2}}+x^{\frac{1}{4}} n^{\frac{1}{2}}\right)\right) . \\
\times \tau^{3}(q)(q, a)^{\frac{1}{2}} \log x \log M .
\end{gathered}
$$

However for any $y \geq 1$ we have

$$
\sum_{n>y} \frac{(q, n)^{\frac{1}{2}}}{n^{2}} \leq \sum_{\delta \mid q} \delta^{\frac{1}{2}} \sum_{\substack{n>y \\ n \equiv 0(\delta)}} \frac{1}{n^{2}} \leq \sum_{\substack{\delta \mid q \\ \delta \leq y}} \delta^{-\frac{3}{2}} \sum_{n>\frac{y}{\delta}} \frac{1}{n^{2}}+\sum_{\substack{\delta \mid y \\ \delta>y}} \delta^{-\frac{3}{2}} \ll y^{-1} \tau(q)
$$

and similarly

$$
\sum_{n>y} \frac{(q, n)^{\frac{1}{2}}}{n^{\frac{3}{2}}} \ll y^{-\frac{1}{2}} \tau(q) .
$$

From (66), (69) - (71) it follows that

$$
S_{1}^{(1)} \ll\left(x^{\frac{1}{2}} M^{-1}+q^{\frac{1}{2}}+x^{\frac{1}{4}} M^{\frac{1}{2}}\right) \tau^{4}(q)(q, a)^{\frac{1}{2}} \log x \log ^{2} M .
$$

We choose $M=\left[x^{\frac{1}{6}}\right]$ and obtain

$$
S_{1}^{(1)} \ll\left(q^{\frac{1}{2}}+x^{\frac{1}{3}}\right) \tau^{4}(q)(q, a)^{\frac{1}{2}} \log ^{4} x .
$$




\subsection{Evaluation of the sums $S_{1}^{(0)}, S_{1}^{(2)} S_{2}^{(0)}, S_{2}^{(1)}$.}

\subsubsection{The sum $S_{1}^{(0)}$.}

We use (15) and (21) to write the sum $S_{1}^{(0)}$ defined by (27) in the form

$$
S_{1}^{(0)}=\sum_{u \leq \sqrt{x / 2}} b_{u} \sqrt{x-u^{2}}, \quad b_{u}=\omega_{a-u^{2}}(q)
$$

and then apply Abel's transformation to get

$$
S_{1}^{(0)}=\sqrt{x / 2} \sum_{u \leq \sqrt{x / 2}} b_{u}-\int_{0}^{\sqrt{x / 2}}\left(\sum_{u \leq t} b_{u}\right) \frac{d}{d t} \sqrt{x-t^{2}} d t .
$$

According to (21) and (25) we have

$$
\sum_{u \leq t} b_{u}=\sum_{\alpha, \beta} \sum_{\substack{u \leq t \\ u \equiv \alpha(q)}} 1=\sum_{\alpha, \beta}\left(\frac{t}{q}+\rho\left(\frac{t-\alpha}{q}\right)-\rho\left(\frac{-\alpha}{q}\right)\right),
$$

hence after certain simple calculations which we leave to the reader and using (3) , (21) and (73) we find that

$$
S_{1}^{(0)}=\left(\frac{\pi}{8}+\frac{1}{4}\right) \frac{\eta_{a}(q)}{q} x+\sqrt{x / 2} \mathfrak{N}-\sqrt{x} \mathfrak{N}_{0}+\sum_{\alpha, \beta} \Gamma_{\alpha},
$$

where

$$
\mathfrak{N}=\sum_{\alpha, \beta} \rho\left(\frac{\sqrt{x / 2}-\alpha}{q}\right), \quad \mathfrak{N}_{0}=\sum_{\alpha, \beta} \rho\left(\frac{-\alpha}{q}\right)
$$

and

$$
\Gamma_{\alpha}=\int_{0}^{\sqrt{x / 2}} \rho\left(\frac{t-\alpha}{q}\right) \frac{t d t}{\sqrt{x-t^{2}}} .
$$

First we prove that

$$
\mathfrak{N}_{0}=\frac{1}{2} \omega_{a}(q)
$$

Using (21) and (75) we can write

$$
\mathfrak{N}_{0}=\sum_{\beta(q)} \sum_{\substack{1 \leq \alpha \leq q \\ \alpha^{2} \equiv a-\beta^{2}(q)}} \rho\left(\frac{-\alpha}{q}\right)=\sum_{\beta(q)} \mathcal{Y}_{\beta}
$$


say.

Consider the sum $\mathcal{Y}_{\beta}$. If $\beta^{2} \not \equiv a(q)$ then we have $\mathcal{Y}_{\beta}=0$. Indeed, in this case there is no term corresponding to $\alpha=q$; the term corresponding to $\alpha=q / 2$ (if such exists) is equal to zero and the other terms can be divided into couples $\rho\left(\frac{-\alpha}{q}\right)+\rho\left(\frac{\alpha-q}{q}\right)$, where $1 \leq \alpha<q / 2$ and the sum of the terms of each such couple equals zero.

If $\beta^{2} \equiv a(q)$ then we have $\mathcal{Y}_{\beta}=\frac{1}{2}$. Indeed, arguing as above we see that the contribution to $\mathcal{Y}_{\beta}$ from the terms corresponding to $1 \leq \alpha<q$ vanishes. In the present case however there is a term corresponding to $\alpha=q$ and its contribution equals $\frac{1}{2}$. This proves (77).

We note that the above arguments imply also

$$
\left|\sum_{\alpha, \beta} \rho\left(\frac{-\alpha}{q}\right) \xi_{\beta}\right| \leq \omega_{a}(q) \quad \text { for } \quad\left|\xi_{\beta}\right| \leq 1
$$

and

$$
\sum_{\alpha, \beta} \sin \left(\frac{2 \pi n \alpha}{q}\right)=0
$$

Consider now the integral $\Gamma_{\alpha}$. The function $\rho(y)$ defined by (24) satisfies

$$
\rho(y)=\sum_{n=1}^{\infty} \frac{\sin (2 \pi n y)}{\pi n} \quad \text { for } \quad y \notin \mathbb{Z} .
$$

We insert this expression in (76) and change the order of summation and integration (here we appeal to the dominated convergence theorem) and we find

$$
\Gamma_{\alpha}=\sum_{n=1}^{\infty} \frac{1}{\pi n} \int_{0}^{\sqrt{x / 2}} \sin \left(2 \pi n \frac{t-\alpha}{q}\right) \frac{t d t}{\sqrt{x-t^{2}}}
$$

Hence using (80) we get

$$
\sum_{\alpha, \beta} \Gamma_{\alpha}=\sum_{n=1}^{\infty} \frac{1}{\pi n} \mathcal{D}_{n} \mathcal{E}_{n}
$$

where

$$
\mathcal{D}_{n}=\sum_{\alpha, \beta} \cos \left(\frac{2 \pi n \alpha}{q}\right), \quad \mathcal{E}_{n}=\int_{0}^{\sqrt{x / 2}} \sin \left(2 \pi n \frac{t}{q}\right) \frac{t d t}{\sqrt{x-t^{2}}}
$$


From (44) and (49) we find

$$
\mathcal{D}_{n} \ll q^{\frac{1}{2}} \tau^{2}(q)(q, n)^{\frac{1}{2}}(q, a)^{\frac{1}{2}}
$$

and integrating by parts we find that

$$
\mathcal{E}_{n} \ll \frac{q}{n} .
$$

Hence applying (70) (with $y=1$ ) and using (81) - (83) we obtain

$$
\sum_{\alpha, \beta} \Gamma_{\alpha} \ll q^{\frac{3}{2}} \tau^{3}(q)(q, a)^{\frac{1}{2}} .
$$

From (74), (77) and (84) we obtain

$$
S_{1}^{(0)}=\left(\frac{\pi}{8}+\frac{1}{4}\right) \frac{\eta_{a}(q)}{q} x+\sqrt{x / 2} \mathfrak{N}-\frac{\sqrt{x}}{2} \omega_{a}(q)+O\left(q^{\frac{3}{2}} \tau^{3}(q)(q, a)^{\frac{1}{2}}\right) .
$$

We note that there is no need to study the sum $\mathfrak{N}$ because in the final expression for $S_{q, a}(x)$ the terms including it cancel each other.

\subsubsection{The sum $S_{1}^{(2)}$.}

From (16), (25), (29), (177) and (79) we easily find

$$
\begin{aligned}
S_{1}^{(2)} & =\sum_{\alpha, \beta} \rho\left(\frac{-\beta}{q}\right)\left(\frac{\sqrt{x / 2}}{q}+\rho\left(\frac{\sqrt{x / 2}-\alpha}{q}\right)-\rho\left(\frac{-\alpha}{q}\right)\right) \\
& =\frac{\omega_{a}(q)}{2 q} \sqrt{x / 2}+O\left((q, a)^{\frac{1}{2}} \tau(q)\right) .
\end{aligned}
$$

\subsubsection{The sum $S_{2}^{(0)}$.}

Using (3), (16), (21), (25), (31), (175) and (177) we find

$$
\begin{aligned}
S_{2}^{(0)} & =\sum_{\alpha, \beta}\left(\frac{\sqrt{x / 2}}{q}+\rho\left(\frac{\sqrt{x / 2}-\alpha}{q}\right)-\rho\left(\frac{-\alpha}{q}\right)\right) \\
& =\frac{\eta_{a}(q)}{q} \sqrt{x / 2}+\mathfrak{N}-\frac{1}{2} \omega_{a}(q) .
\end{aligned}
$$




\subsubsection{The sum $S_{2}^{(1)}$.}

From (16), (25), (32), (75) and (79) we get

$$
\begin{aligned}
S_{2}^{(1)} & =\sum_{\alpha, \beta} \rho\left(\frac{\sqrt{x / 2}-\beta}{q}\right)\left(\frac{\sqrt{x / 2}}{q}+\rho\left(\frac{\sqrt{x / 2}-\alpha}{q}\right)-\rho\left(\frac{-\alpha}{q}\right)\right) \\
& =\frac{\sqrt{x / 2}}{q} \mathfrak{N}+\mathfrak{D}+O\left((q, a)^{\frac{1}{2}} \tau(q)\right),
\end{aligned}
$$

where

$$
\mathfrak{D}=\sum_{\alpha, \beta} \rho\left(\frac{\sqrt{x / 2}-\alpha}{q}\right) \rho\left(\frac{\sqrt{x / 2}-\beta}{q}\right) .
$$

Consider the sum $\mathfrak{D}$. We take an integer $M_{1} \geq 2$ and apply (34) with $M=M_{1}$ to get

$$
\mathfrak{D}=\sum_{\alpha, \beta} \rho\left(\frac{\sqrt{x / 2}-\alpha}{q}\right) \sum_{1 \leq|n| \leq M_{1}} \frac{1}{2 \pi i n} e\left(\frac{\sqrt{x / 2}-\beta}{q} n\right)+O\left(\Delta_{1}\right),
$$

where

$$
\Delta_{1}=\sum_{\alpha, \beta} \min \left(1, M_{1}^{-1}\left\|\frac{\sqrt{x / 2}-\beta}{q}\right\|^{-1}\right) .
$$

Applying (34) again and having in mind (44) and (90) we find

$$
\begin{gathered}
\mathfrak{D}=\sum_{\alpha, \beta} \sum_{1 \leq|m| \leq M_{1}} \frac{1}{2 \pi i m} e\left(\frac{\sqrt{x / 2}-\alpha}{q} m\right) \sum_{1 \leq|n| \leq M_{1}} \frac{1}{2 \pi i n} e\left(\frac{\sqrt{x / 2}-\beta}{q} n\right) \\
+O\left(\Delta_{1} \log M_{1}\right) \\
=\sum_{1 \leq|m|,|n| \leq M_{1}} \frac{e\left((m+n) q^{-1} \sqrt{x / 2}\right)}{(2 \pi i)^{2} m n} \mathcal{H}_{m, n}+O\left(\Delta_{1} \log M_{1}\right) .
\end{gathered}
$$

Next we use (35) (with $M=M_{1}$ ) to write the sum $\Delta_{1}$ defined by (90) in the form

$$
\Delta_{1}=\sum_{\alpha, \beta} \sum_{n \in \mathbb{Z}} c_{n} e\left(\frac{\sqrt{x / 2}-\beta}{q} n\right)=\sum_{n \in \mathbb{Z}} c_{n} e\left(\frac{\sqrt{x / 2}}{q} n\right) \mathcal{H}_{0, n} .
$$


From (3), (36), (49), (66), (70), (91) and (92) we find

$$
\begin{gathered}
\mathfrak{D} \ll \sum_{1 \leq|m|,|n| \leq M_{1}} \frac{\left|\mathcal{H}_{m, n}\right|}{|m n|}+\log ^{2} M_{1}\left(\frac{\eta_{a}(q)}{M_{1}}+\sum_{1 \leq|n| \leq M_{1}} \frac{\left|\mathcal{H}_{0, n}\right|}{|n|}+M_{1} \sum_{|n|>M_{1}} \frac{\left|\mathcal{H}_{0, n}\right|}{n^{2}}\right) . \\
\ll q^{\frac{1}{2}} \tau^{2}(q)(q, a)^{\frac{1}{2}}\left(\sum_{1 \leq m, n \leq M_{1}} \frac{(q, m, n)^{\frac{1}{2}}}{m n}+\sum_{1 \leq n \leq M_{1}} \frac{(q, n)^{\frac{1}{2}}}{n}+M_{1} \sum_{n>M_{1}} \frac{(q, n)^{\frac{1}{2}}}{n^{2}}\right) \log ^{2} M_{1} \\
+\frac{\log ^{2} M_{1}}{M_{1}} \eta_{a}(q) \\
\ll q^{\frac{1}{2}} \tau^{3}(q)(q, a)^{\frac{1}{2}} \log ^{4} M_{1}+\frac{\log ^{2} M_{1}}{M_{1}} \eta_{a}(q) .
\end{gathered}
$$

Now we choose $M_{1}=q^{2}$ and having in mind (3) we get

$$
\mathfrak{D} \ll q^{\frac{1}{2}} \tau^{3}(q)(q, a)^{\frac{1}{2}} \log ^{4} x .
$$

From (88) and (93) we obtain

$$
S_{2}^{(1)}=\frac{\sqrt{x / 2}}{q} \mathfrak{N}+O\left(q^{\frac{1}{2}} \tau^{3}(q)(q, a)^{\frac{1}{2}} \log ^{4} x\right)
$$

\subsection{The end of the proof.}

It remains to collect together (33), (72), (85), (86), (87) and (94) and we establish (10), which proves the theorem.

\section{References}

[1] E. Bombieri, On exponential sums in finite fields, Amer. J. Math., 88, 1, (1966), 71-105.

[2] V. Blomer, The average value of divisor sums in arithmetic progressions, Quart. J. Math. 59 (2008), 275-286.

[3] V. Blomer, J. Brüdern, R. Dietmann, Sums of smooth squares, Compos. Math. 145 (2009), 1401-1441. 
[4] T. Esterman, A new application of the Hardi-Littlewood-Kloosterman method, Proc. London Math. Soc., 12 (1962), 425-444.

[5] S. W. Graham, G. Kolesnik, Van der Corput method of exponential sums, Cambridge Univ. Press, 1991.

[6] C. Hooley, Applications of sieve methods to the theory of numbers, Cambridge Univ. Press, 1976.

[7] L. K. Hua, Introduction to number theory, Springer, 1982.

[8] H. Iwaniec, E. Kowalski, Analytic number theory, Colloquium Publications, vol. 53, Amer. Math. Soc., 2004.

[9] R. A. Smith, The circle problem in an arithmetic progression, Canad. Math. Bull., 11, 2, (1968), 175-184.

[10] A. V. Ustinov, On the number of solutions of the congruence $x y \equiv 1(\bmod q)$ under the graph of a twice continuously differentiable function, (In Russian), Algebra and Analysis, 20, 5, (2008), 186-216.

[11] P. D. Varbanets, Lattice points in a circle whose distances from the center are in an arithmetic progression, (In Russian), Matem. Zametki, 8, 6, (1970), 787-798.

Faculty of Mathematics and Informatics

Sofia University "St. Kl. Ohridsky"

5 J.Bourchier, 1164 Sofia, Bulgaria

Email: dtolev@fmi.uni-sofia.bg 